\title{
Surface-functionalised nano-beads as novel supports for organic synthesis
}

\author{
Andrew N. Cammidge, ${ }^{*}$ Stuart Downing and Zainab Ngaini \\ Wolfson Materials and Catalysis Centre, School of Chemical Sciences and Pharmacy, University of East Anglia, \\ Norwich NR4 7TJ, UK
}

Received 14 May 2003; revised 23 June 2003; accepted 3 July 2003

\begin{abstract}
A novel polymer support has been prepared in which functional link points are located on the surface of polymer nano-beads; the use of the support has been demonstrated in the syntheses of unsymmetrical porphyrins.

(C) 2003 Elsevier Ltd. All rights reserved.
\end{abstract}

The use of solid-phase supports in organic synthesis is now widely employed. ${ }^{1}$ Although inorganic supports such as silica gels and controlled pore glasses ${ }^{2}$ are frequently used, organic polymers still comprise the largest class of support materials. Lightly crosslinked, swellable polymers are typically prepared by emulsion polymerisation techniques to give beads in sizes ranging from $\mu \mathrm{m}$ to $\mathrm{mm}$. Such insoluble polymers are convenient for application as supports for synthesis, reagents, scavengers etc. Their efficiency is governed by their ability to swell (allowing access through the polymer matrix) and therefore requires the use of suitable solvents. This limitation is usually accommodated but complications can be envisaged when attempts are made to build up large molecules within the pores of such supports.

In this letter we describe the preparation of a new type of polymeric support based on surface-functionalised nanoparticles. We envisaged that an ideal solid support could comprise small solid particles (ca. 50-300 nm) bearing link points exclusively at the surface. Such supports would be soluble in reaction media but easily isolated by precipitation or SEC. Locating the link points at the surface obviates the problems associated with 'buried' functionality within a porous polymer (heterogeneity of sites, accessibility etc).

We reasoned that such a support could be realised via polymerisation of a microemulsion ${ }^{3}$ in which a polymerisable sufactant serves as amphiphile and, following

\footnotetext{
* Corresponding author.
}

polymerisation, as functional link point for solid-phase organic synthesis. We chose a polymerisable carboxylic acid derivative as surfactant to ensure a useful link group would be attached to the final polymer (reasoning that the carboxylic acid could be reduced to provide additional scope). Initial experiments with simple 'surfactants' such as 4-vinylbenzoic acid were unsuccessful and led to the choice of amphiphile $4 .^{4}$ Compound $\mathbf{4}$ was most conveniently synthesised by the route depicted in Scheme 1.

In a typical procedure polymerisable amphiphile 4 (1.2 g) and CTAB (6.8 g) were used as surfactants to stabilise a styrene/divinylbenzene $(10: 1,2.4 \mathrm{~g})$ microemulsion in water $(189 \mathrm{~mL})$. The microemulsion was polymerised (AIBN initiation) at $60^{\circ} \mathrm{C}$ for $7 \mathrm{~h}$ to give a transparent solution of the surface-functionalised nanoparticles. Acidification and addition of methanol precipitated the polymer from solution and it was filtered off and washed repeatedly with water to remove CTAB. The resulting polymer was dried under vacuum and characterised by TEM (average bead diameter 317 $\left.\mathrm{nm}^{5}\right)$, IR spectroscopy $\left(v_{\mathrm{C}=\mathrm{O}} 1707 \mathrm{~cm}^{-1}\right)$ and titration (loading $=0.89 \mathrm{mmol} / \mathrm{g} \mathrm{COOH}$ ) (Scheme 2).

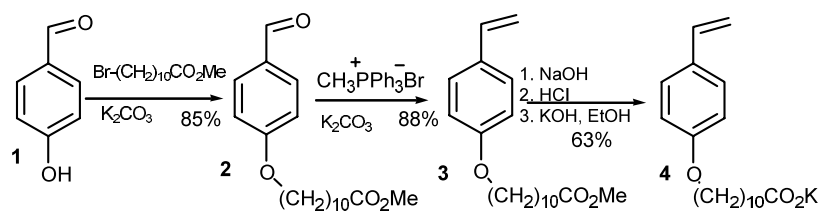

Scheme 1. Synthesis of 4 . 